\title{
Influence of Thermal Annealing Media on Optical and Electrical Properties of FTO, ITO and $\mathrm{TiO}_{2}$ Films
}

\author{
Tien Thanh Nguyen ${ }^{1,4, *}$, Khac An Dao ${ }^{2,3,4, *}$, Thi Thuy Nguyen ${ }^{4}$, Chung Dong Nguyen ${ }^{4}$, \\ Si Hieu Nguyen ${ }^{4}$ and Thi Mai Huong Nguyen ${ }^{5}$ \\ ${ }^{1}$ Graduate University of Science and Technology, Vietnam Academy of Science and Technology (VAST), 18 Hoang Quoc Viet, Cau Giay, \\ HaNoi 100000, Vietnam \\ ${ }^{2}$ Institute of Theoretical and Applied Research, Duy Tan University, HaNoi 100000, Vietnam \\ ${ }^{3}$ Faculty of Electrical-Electronic Engineering, Duy Tan University, Da Nang 550000, Vietnam \\ ${ }^{4}$ Institute of Materials Science, VAST, 18 Hoang Quoc Viet, Cau Giay, HaNoi 100000, Vietnam \\ ${ }^{5}$ Institute of Applied Physics and Scientific Instruments, VAST, 18 Hoang Quoc Viet, Cau Giay, HaNoi 100000, Vietnam
}

\begin{abstract}
Metal oxides, in many cases, exhibit n-type semiconductors due to the existence of oxygen vacancies in the lattice. Therefore, the interactions of oxygen being in medium with oxygen vacancies during the annealing process can change concentrations of defects that will cause variations in optical and electric properties of such materials. However, research on such interactions for commercial FTO, ITO, and TiO 2 products has been limited. This paper summarizes the results of some experiments conducted to determine the influence of thermal annealing media on the optical, electrical properties of thin films of these products. The thermal media considered are air medium, $10^{-1}$ torr low vacuum condition, and Argon gas environments, and the annealing condition is set at $450^{\circ} \mathrm{C}$ for 20 minutes. It is found that while FTO films change for the better after annealing, ITO films trend towards worse, and $\mathrm{TiO}_{2}$ films have the most photoconversion efficiency $(I s c=0.27 \mathrm{~mA}, \eta=0.37 \%)$ under the moderate oxygen concentration environment. [doi:10.2320/matertrans.MT-MN2019002]
\end{abstract}

(Received November 15, 2019; Accepted August 3, 2020; Published October 2, 2020)

Keywords: solar cells, $\mathrm{TiO}_{2}, \mathrm{FTO}$, ITO thin films, influence of annealing condition, oxygen vacancies

\section{Introduction}

Fluorine doped Tin Oxide (FTO), Indium Tin Oxide (ITO), and Titanium Dioxide $\left(\mathrm{TiO}_{2}\right)$ are principal metal oxides that are used most widely, especially in the fields of the solar cell, light-emitting diode, photocatalyst, water splitting, and sensor. FTO and ITO films are also used as transparent electrodes, so they have to meet the two vital requirements, namely high transmittance and low resistance. Tin Oxide $\left(\mathrm{SnO}_{2}\right)$ is an n-type semiconductor whose conductivity is resulted mainly from the contribution of oxygen vacancies $\left(\mathrm{V}_{\mathrm{O}}\right)$ in the crystal lattice, and it can be increased through doping by elements in groups III, V, VI, VII. ${ }^{1-3)}$ Among them, Fluorine $(\mathrm{F})$ is most widely used to replace Oxygen atoms $(\mathrm{O})$ in the $\mathrm{SnO}_{2}$ lattice due to their similar anion size of 1.32 and $1.33 \AA$ for $\mathrm{F}^{-}$and $\mathrm{O}^{2-}$ ions, respectively. ${ }^{1,4)}$ The hybrid orbital configuration of $\mathrm{F}$ and $\mathrm{O}$ is $2 \mathrm{~s}^{2} 2 \mathrm{p}^{5}$ and $2 \mathrm{~s}^{2} 2 \mathrm{p}^{4}$, respectively, which indicates that the $\mathrm{F}$ atom promotes one electron per one molecule when it replaces $\mathrm{O}$ atom and thus acting as an electron donor level which leads to the n-type conduction mechanism. ${ }^{5,6)}$ In the case of ITO thin films, the Indium Oxide $\left(\mathrm{In}_{2} \mathrm{O}_{3}\right)$-based materials are doped with Tin (Sn) to increase conductivity, ${ }^{7,8)}$ and $\mathrm{Sn}$ acts as a cationic dopant replacing Indium (In) to bind with $\mathrm{O}$ in the plane. With valence of 3 and 4 for In and Sn, respectively, the replacement forms a donor level near the conduction band. ${ }^{7,8)}$ Both FTO and ITO have high conductivity because of their high carrier concentration generated by the two mechanisms. The first is the replacement of the $\mathrm{F}$ atom for the $\mathrm{O}$ atom for FTO and the replacement of the In atom by the Sn atom in

*Corresponding authors, E-mail: ntthanh@ims.vast.ac.vn, andaokhac@ gmail.com
ITO, resulting in an excess electron. The second one is the natural existence of $\mathrm{V}_{\mathrm{O}}$ in the metal oxide, which forms a donor level for two electrons. ${ }^{1-8)}$

$\mathrm{TiO}_{2}$ is a favorite material used as an electron transport layer in optoelectronic devices because of its superior properties. In stoichiometric $\mathrm{TiO}_{2}$, each $\mathrm{Ti}$ atom gives four electrons from $4 \mathrm{~s}$ and $3 \mathrm{~d}$ orbitals to two $\mathrm{O}$ atoms $(2 \mathrm{p}$ orbital). ${ }^{9-11)}$ For this reason, $\mathrm{Ti}^{4+}$ ions have an electronic configuration of $3 \mathrm{~d}^{0}$ that forms the empty conducting bands of pure $\mathrm{TiO}_{2}$ materials. The physical and chemical properties of $\mathrm{TiO}_{2}$ nanomaterials depend on the presence of various crystalline defects. Among them, $\mathrm{V}_{\mathrm{O}}$ has the lowest formation energy but plays a vital role in determining material characteristics, which is of great interest to both theoretical and experimental researchers. ${ }^{9)}$

Vacancy defects, which are generated by the absence of atoms/ions in the lattice structure, are common concepts in all types of crystalline materials..$^{9,12-16)}$ They are point defects formed to minimize Helmholtz's free energy to establish thermodynamic equilibrium at a specific temperature. There are two types of vacancy defects: a negative ion defect (or anion defect), and a positive ion defect (or cation defect). ${ }^{9}{ }^{17)} \mathrm{V}_{\mathrm{O}}$ is an anion vacancy that can be adjusted by heat treatment techniques in different environments. ${ }^{9,18-20)}$

Because of the extensive range of applications, FTO, ITO, and $\mathrm{TiO}_{2}$ have become readily available for commercial purposes. It is sometimes required to anneal ITO, FTO, $\mathrm{TiO}_{2}$ films within the process of using them (to crystallize materials, remove additional organic materials, and increase the mechanical strength of the films). However, up to the present, no reports have been found on detailed investigations of the dependence of the optical and electrical properties of these commercial products on the annealed conditions. 
Therefore, an investigation in more detail is made in this report to better understand the effect of different annealed environments on the optical and electrical properties of FTO, ITO, and $\mathrm{TiO}_{2}$ films. The obtained experimental results are outlined in the next section, followed by further discussions.

\section{Experimental Procedure}

FTO, ITO films on glass, and $\mathrm{TiO}_{2}$ powder (P25) are commercial products ordered from Sigma-Aldrich, and $\mathrm{TiO}_{2}$ films are prepared from this powder through the two following steps. The first is to diffuse P25 into ethanol to form a $1.8 \%$ solution (weight percentage). The second is spin-coating processing set at the rate of $3000 \mathrm{rpm}$ in 30 seconds to make $\mathrm{TiO}_{2}$ films on FTO substrate from the above solution, and this process is implemented through a required number of deposition times to achieve the thickness of the film of about $1000 \mathrm{~nm}$. After $\mathrm{TiO}_{2}$ films were already prepared, the annealing process for FTO, ITO, and $\mathrm{TiO}_{2}$ films was done at $450^{\circ} \mathrm{C}$ in air, low vacuum, and Argon gas media for 20 minutes (Table 1 shows the designation of samples under different annealing conditions). Interactions between $\mathrm{V}_{\mathrm{O}}$ and oxygen in the medium process followed the reaction below:

$$
V_{O}+\frac{1}{2} O_{2} \rightleftharpoons O^{2-}-2 e^{-}
$$

where $\mathrm{O}_{2}$ is a molecule from the environment and $\mathrm{O}^{2-}$ is an ion in the lattice.

The concentration of $\mathrm{V}_{\mathrm{O}}\left[V_{O}\right]$ depends on the partial pressure of $\mathrm{O}\left(P_{O}\right)$ at a specified temperature according to the following rule: ${ }^{18)}$

$$
\left[V_{O}\right]=C(T) P_{O}^{-1 / m}
$$

where $C(T)$ is a function of temperature, and $m$ is a constant.

It has been reported that $m=6$ or $m=2$ for $\mathrm{TiO}_{2}{ }^{18)}$ In our experimental case, the low vacuum environment has an Oxygen partial pressure which is smaller than the air environment but greater than the Ar gas environment. Therefore, the Oxygen vacancies concentration of the thin films annealed in Ar gas medium is highest, followed by those in the vacuum, and is lowest in the air atmosphere.

The optical of the films was measured by Agilent Cary 5000 spectrometer, and the sheet resistance of the films is done by the four-probe method. Besides, investigating the photoelectrochemical characteristics of the $\mathrm{TiO}_{2}$ films is carried out through a three-electrode configuration. In this investigation, all of the system components, including $\mathrm{TiO}_{2}$ films as photoelectrodes, $\mathrm{Pt}$ as a counter electrode, and $\mathrm{Ag}$ / $\mathrm{AgCl}$ as a reference electrode were immersed in the $\mathrm{Kpi}$ buffer solution $(\mathrm{pH}=7)$ and under one-sun illumination (AM 1.5, $100 \mathrm{~mW} / \mathrm{cm}^{2}$ ).

Table 1 Designation of samples under different annealing conditions.

\begin{tabular}{lccr}
\hline Conditions & ITO & FTO & TiO2 \\
\hline Not annealed & I-0 & F-0 & \\
Annealed in Argon gas & I-Ar & F-Ar & T-Ar \\
Annealed in Vacuum & I-Va & F-Va & T-Va \\
Annealed in Air & I-Am & F-Am & T-Am \\
\hline
\end{tabular}

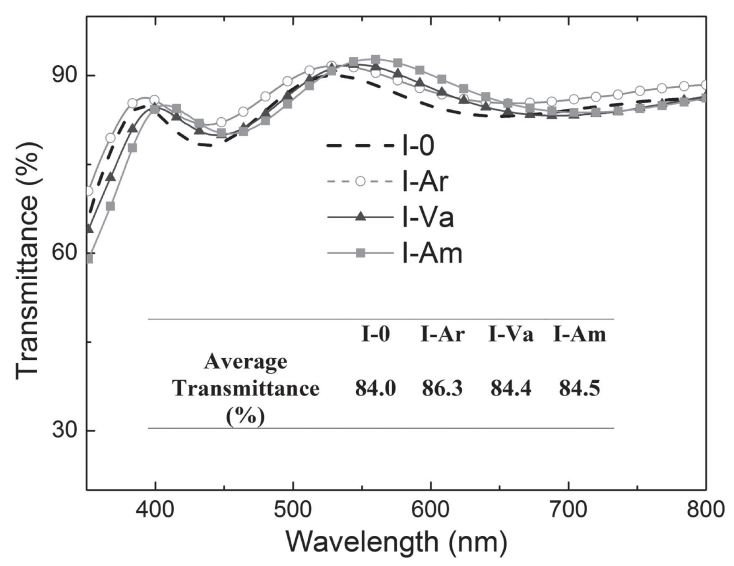

Fig. 1 The transmission spectra of ITO films when annealing under different conditions.

\section{Results and Discussions}

\subsection{The effect on the transmittance and sheet resistance of ITO and FTO films}

The transmittance spectra of the ITO films under different annealing conditions are shown in Fig. 1. It can be seen that the transmittances are more or less the same for all samples; namely, the average transmittance taken as the average of the transmittance within the range of $350-800 \mathrm{~nm}$ is about $84-86 \%$. The oscillation of the transmittance spectra like the seen in the figure probably comes from the light interference phenomena between the ITO films and the substrate (glass). While their transmittance is not affected by the annealing media, these sheet resistance strongly depend on it. The sheet resistance values of ITO films are shown in Fig. 3, the film annealed in the air medium has four times greater resistance than that of the film not annealed $(26.53 \Omega$ compared to $6.27 \Omega$ ) whereas the film annealed in Argon gas medium has negligible small resistance change $(8.72 \Omega$ versus $6.27 \Omega$ ). This can be explained by the fact that the annealing environment and temperature could considerably affect the free electron concentration of ITO films. ${ }^{21)}$ ITO is an n-type semiconductor with electron donor levels formed by both the doping process of $\mathrm{Sn}$ into the $\mathrm{In}_{2} \mathrm{O}_{3}$ lattice structure and the existence of $\mathrm{V}_{\mathrm{O}}$ in the material. The $\mathrm{V}_{\mathrm{O}}$ concentration was decreased due to interacting with oxygen in the annealing environment and the free electron concentration decreases following the decrease of the $\mathrm{V}_{\mathrm{O}}$.

In Fig. 2, the transmittance spectra of FTO films are shown, and the average transmittance calculated in a range of $350 \mathrm{~nm}$ to $800 \mathrm{~nm}$. It can be seen that the annealing medium also does not affect the transmittance of FTO films (about $86 \%$ for all annealed films), and it is slightly better than non-annealed films ( $86 \%$ versus $81 \%$ ). This may be due to better crystallization of the films after annealing, thereby reducing scattering and increasing transmittance. The sheet resistance is about $14 \Omega$ /sq for all FTO films under different annealing conditions (see Fig. 3), and they were not affected by the annealing condition due to the low $\mathrm{V}_{\mathrm{O}}$ concentration of FTO films. $\mathrm{V}_{\mathrm{O}}$ concentration of FTO film is much lower than that of $\mathrm{SnO}_{2}$ and ITO because $\mathrm{F}$ is anion dopant that can either replace interstitial $\mathrm{O}$ or occupy $\mathrm{V}_{\mathrm{O}}$ existed formerly in the $\mathrm{SnO}_{2}$ crystal. Occupying $\mathrm{V}_{\mathrm{O}}$ may be easier than 


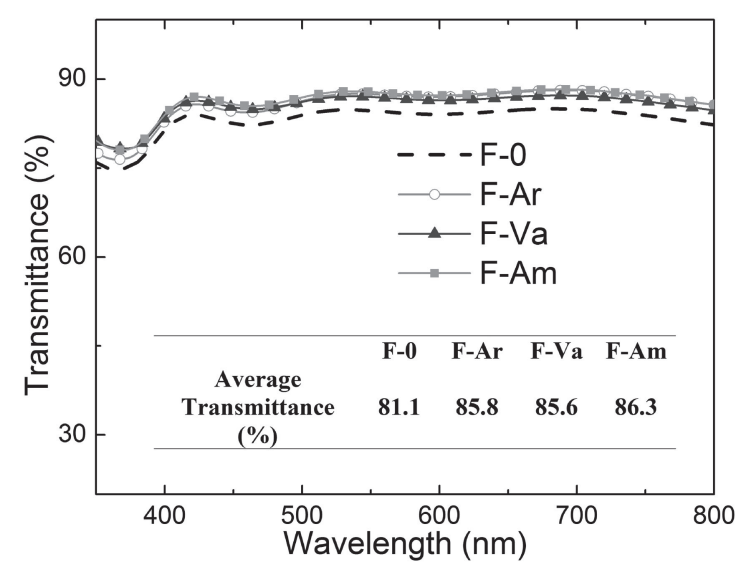

Fig. 2 The transmission spectra of FTO films when annealing under different conditions.

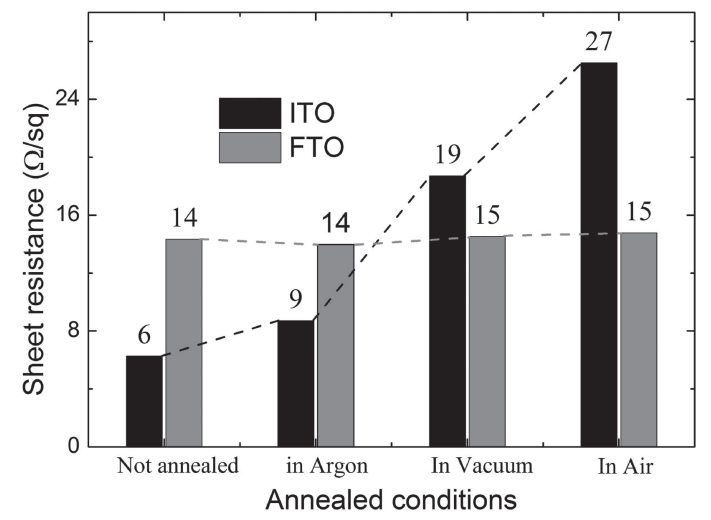

Fig. 3 Comparison of sheet resistances of ITO and FTO films under different annealing conditions.

replacing $\mathrm{O}$ atoms because lower energy requirements lead to a decrease in $\mathrm{V}_{\mathrm{O}}$ concentration in FTO films.

\subsection{Comparison of ITO and FTO films}

The transmittance spectra, illustrated in Figs. 1 and 2, of all ITO and FTO films, reveal that the FTO films have a better transmittance than the ITO films in the wavelength range of $400-500 \mathrm{~nm}$. However, ITO films behave better within the wavelength range of 500-600 $\mathrm{nm}$. Therefore, their average transmittance is not much different (about 84-86\%). On the contrary, the charts shown in Fig. 3 confirm that sheet resistance of FTO films is the same, but the results are entirely different in the case of ITO films. It is worth noting that in the absence of annealing, the ITO film has a much smaller resistance than the FTO film $(6 \Omega$ and $14 \Omega$, respectively). This result could be explained by the fact that the free electron concentration in the conduction band of ITO is higher than FTO. The reasons could be the following: (i) the number of $\mathrm{V}_{\mathrm{O}}$ of ITO is higher FTO and (ii) The solubility of $\mathrm{Sn}$ in $\mathrm{In}_{2} \mathrm{O}_{3}$ is better than that of $\mathrm{F}$ in $\mathrm{SnO}_{2}{ }^{7}$ ) When the films are annealed in argon gas medium, the resistance of the ITO film is still smaller FTO film $(9 \Omega$ compared to $14 \Omega$ ). However, when they are annealed in the low vacuum, the ITO film resistance is higher $(19 \Omega$ compared to $15 \Omega$ ) and almost twice higher if they are annealed in air medium $(27 \Omega$ versus $15 \Omega)$. These results suggest that it should use ITO film as an electrode in case of

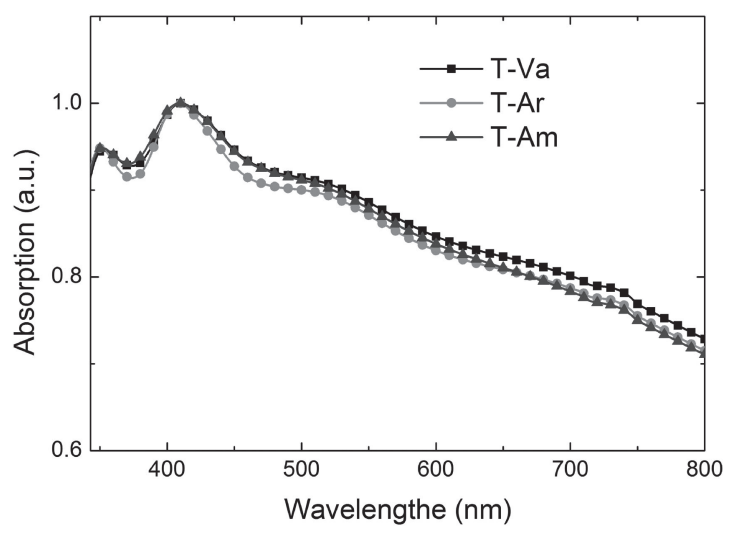

Fig. 4 The absorption spectra of $\mathrm{TiO}_{2}$ films under different annealing conditions.

without annealing or annealing in inert gas. In contrast to this, FTO films should be better for that purpose.

\subsection{The effect on the optical and electrical properties of $\mathrm{TiO}_{2}$ film}

Figure 4 shows the absorption spectra of $\mathrm{TiO}_{2}$ films annealed in different conditions. Three absorption peaks can observe correspond to the three electron transition mechanisms from the valence band to higher levels. Generally, the various annealing mediums did not make a change in these absorption spectra but create a difference in electron characteristics of the $\mathrm{TiO}_{2}$ films. Figures 5(a) and 5(b) show the linear sweep voltammetry (LSV) and photoconversion efficiency spectra, respectively, of $\mathrm{TiO}_{2}$ films under different annealing conditions. Although the difference in their optical properties is not significant, as mentioned above, the change in their electrical properties is clearly. Short-circuit current (Isc) achieves the highest value for the T-Va electrode $(0.27 \mathrm{~mA})$ and the lowest for T-Am $(0.12 \mathrm{~mA})$. The difference is 2.25 times, and the corresponding value in the case of their photoconversion efficiency is 2.64 times. It can be verified by the results being in Fig. 5(b) and Table 2. The photoconversion efficiency is highest at $0.37 \%$ for $\mathrm{T}-\mathrm{Va}$, followed by the lowest of $0.14 \%$ for T-Am films. The enhancing of $\mathrm{TiO}_{2}$ film electrical conductivity depends on the increase of the $\mathrm{V}_{\mathrm{O}},{ }^{18,22)}$ thus, the electrical resistivity of T-Am film will be the lowest, but the obtained results shown its photoconversion efficiency is not the highest. A similar effect on $\mathrm{WO}_{3}$ material was published by M. Sachs et al.; ${ }^{14)}$ therefore, it can be concluded that a medium of moderate oxygen concentration becomes the best for the annealing $\mathrm{TiO}_{2}$.

\section{Conclusion}

Metal oxides materials, namely ITO, FTO, and $\mathrm{TiO}_{2}$, are always essential research objects relating to the manufacture of various devices such as solar cells, light-emitting diodes, photocatalysts, water splitting, and sensors. For those materials, defects, especially $\mathrm{V}_{\mathrm{O}}$, play a vital role in determining properties of these materials. It is found through our experiments that the optical characteristics of these three films are not significantly influenced by annealing media. More importantly, the electrical characteristics do 


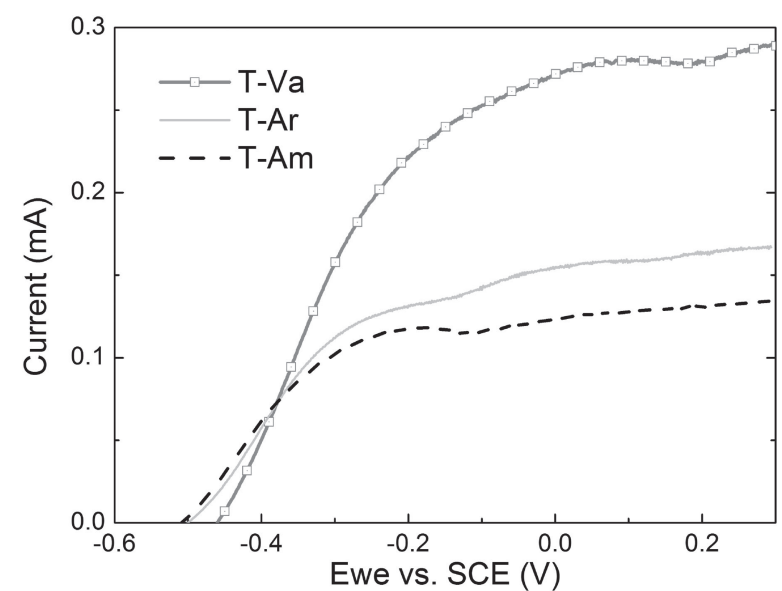

(a)

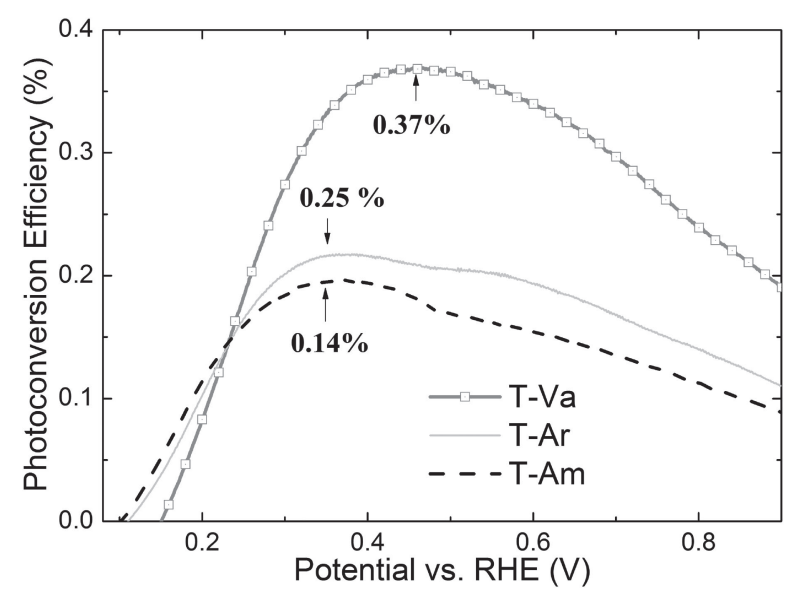

(b)

Fig. 5 The linear sweep voltammetry (a) and photoconversion efficiency (b) spectra of $\mathrm{TiO}_{2}$ films under different annealing conditions.

Table 2 Some photoelectric parameters of the $\mathrm{TiO}_{2}$ films, open circuit potential $\left(\mathrm{V}_{\mathrm{oc}}\right)$, short-circuit current $\left(\mathrm{I}_{\mathrm{sc}}\right)$, and maximum photoelectric conversion efficiency $\left(\eta_{\max }\right)$.

\begin{tabular}{cccc}
\hline Parameters & $\mathrm{T}-\mathrm{Ar}$ & $\mathrm{T}-\mathrm{Va}$ & $\mathrm{T}-\mathrm{Am}$ \\
\hline $\mathrm{V}_{\mathrm{oc}}($ at $\mathrm{I}=0 \mathrm{~mA})$ & $0.492 \mathrm{~V}$ & $0.459 \mathrm{~V}$ & $0.504 \mathrm{~V}$ \\
$\mathrm{I}_{\mathrm{sc}}($ at $\mathrm{V}=0 \mathrm{~V})$ & $0.154 \mathrm{~mA}$ & $0.271 \mathrm{~mA}$ & $0.122 \mathrm{~mA}$ \\
& $0.251 \%$ & $0.372 \%$ & $0.144 \%$ \\
$\eta_{\max }$ & $($ at $\mathrm{V}=0.37 \mathrm{~V})$ & $($ at $\mathrm{V}=0.46 \mathrm{~V})$ & $($ at $\mathrm{V}=0.36 \mathrm{~V})$ \\
\hline
\end{tabular}

vary, depending on both the materials and annealing media. In particular, the electrical properties of all FTO films remain almost unchanged because of the reduction of the number of $\mathrm{V}_{\mathrm{O}}$ by doping of $\mathrm{F}$ atoms. On the contrary, the sheet resistance of ITO film increases significantly with the $\mathrm{P}_{\mathrm{O}}$ in the annealing media. For instance, such the resistance under the air medium annealing increased by 4.5 folds, compared to the case without annealing, due to the relative losses of $\mathrm{V}_{\mathrm{O}}$. In addition, it is also found that among the different environments, the photoelectric performance of $\mathrm{TiO}_{2}$ film under the medium oxygen concentration environment is the best (with $I s c$ and $\eta$ being $0.27 \mathrm{~mA}$ and $0.37 \%$, respectively), which implies the importance of selecting the right annealing conditions to improve photo-electrical performances of devices. Our further study will be continued in more detail in the forthcoming paper.

\section{Acknowledgments}

This research is funded by the Vietnam National Foundation for Science and Technology Development (NAFOSTED) under grant number 103.02-2017.346. The authors would like to express our sincere thanks to the Institute of Materials Science (IMS), Vietnam Academy of Science and Technology (VAST) for their supports and encouragements.

\section{REFERENCES}

1) M.L.M. Napi, M.F. Maarof, C.F. Soon, N. Nayan, F.I.M. Fazli, N.K.A. Hamed, S.M. Mokhtar, N.K. Seng, M.K. Ahmad, A.B. Suriani and A. Mohamed: ARPN J. Eng. Appl. Sci. 11 (2016) 8800.

2) A.V. Moholkar, S.M. Pawar, K.Y. Rajpure, S.N. Almari, P.S. Patil and C.H. Bhosale: Sol. Energy Mater. Sol. Cells 92 (2008) 1439-1444.

3) A.A. Yadav, E.U. Masumdar, A.V. Moholkar, K.Y. Rajpure and C.H. Bhosale: Physica B 404 (2009) 1874-1877.

4) B. Russo and G.Z. Cao: Appl. Phys. A 90 (2007) 311-315.

5) Z. Banyamin, P. Kelly, G. West and J. Boardman: Coatings 4 (2014) 732-746.

6) P.V. Bhuvaneswari, P. Velusamy, R.R. Babu, S.M. Babu, K. Ramamurthi and M. Arivanandhan: Mater. Sci. Semicond. Process. 16 (2013) 1964-1970.

7) S. Ngamsinlapasathian, A. Kitiyanan, T. Fujieda and S. Yoshikawa: ECS Trans. 1(33) (2006) 7-15.

8) B.-S. Chiou and J.-H. Tsai: J. Mater. Sci. Mater. Electron. 10 (1999) $491-495$.

9) A. Sarkar and G.G. Khan: Nanoscale 11 (2019) 3414-3444.

10) E. Cho, S. Han, H.-S. Ahn, H.K.-R. Lee, S.K. Kim and C. Seong: Phys. Rev. B 73 (2006) 193202.

11) C. Lin, D. Shin and A.A. Demkov: J. Appl. Phys. 117 (2015) 225703

12) M.V. Ganduglia-Pirovano, A. Hofmann and J. Sauer: Surf. Sci. Rep. 62 (2007) 219-270.

13) G.L. Kabongo, T.N.Y. Khawula, T. Thokozani, E.G. Nyongombe, K Ozoemena and S. Dhlamini: J. Nanosci. Curr. Res. 3 (2018) 1000125

14) M. Sachs, J.-S. Park, E. Pastor, A. Kafizas, A.A. Wilson, L. Francàs, S. Gul, M. Ling, C. Blackman, J. Yano, A. Walsh and J.R. Durrant: Chem. Sci. 10 (2019) 5667-5677.

15) F. Mehmood, R. Pachter, N.R. Murphy, W.E. Johnson and C.V. Ramana: J. Appl. Phys. 120 (2016) 233105.

16) M.M. El-Nahass, H.S. Soliman and A. El-Denglawey: Appl. Phys. A 122 (2016) 775.

17) J. Wang, Z. Wang, B. Huang, Y. Ma, Y. Liu, X. Qin, X. Zhang and Y. Dai: ACS Appl. Mater. Interfaces 4 (2012) 4024-4030.

18) C.J. Kevane: Phys. Rev. 133 (1964) A1431-A1436.

19) B. Santara, P.K. Giri, K. Imakita and M. Fujii: Nanoscale 5 (2013) 5476.

20) B. Choudhury, S. Bayan, A. Choudhury and P. Chakraborty: J. Colloid Interface Sci. 465 (2016) 1-10.

21) S.J. Hong, J.W. Kim, J.W. Lim, G.S. Choi and M. Isshiki: Mater. Trans. 51 (2010) 1905-1908.

22) Y. Lu, M. Hirohashi and K. Sato: Mater. Trans. 47 (2006) 1449-1452. 